\title{
Reabilitação em Artrite Idiopática Juvenil
}

\section{Rehabilitation in Juvenile Idiopathic Arthritis}

\author{
Vanessa Cristina Bueno( ${ }^{(1)}$, Império Lombardi Júnior ${ }^{(2)}$, Wladimir Musetti Medeiros ${ }^{(3)}$, \\ Mariana Moreira Alckmin Azevedo ${ }^{(4)}$, Cláudio Arnaldo Len ${ }^{(5)}$, Maria Teresa R. A. Terreri ${ }^{(6)}$, \\ Jamil Natour ${ }^{(7)}$, Maria Odete Esteves Hilário ${ }^{(8)}$
}

\section{RESUMO}

Introdução e objetivos: a artrite idiopática juvenil (AIJ) pode acarretar em seu curso clínico incapacidades físicas permanentes em crianças e adolescentes. Este estudo teve como objetivos a descrição das diversas modalidades de reabilitação, desde a avaliação até a prescrição de exercícios, bem como a elaboração de um guia prático de reabilitação para pacientes com AIJ. Fonte de dados: a pesquisa foi realizada nas bases de dados do Medline e do Lilacs. $\mathrm{Na}$ discussão dos diversos tópicos, foi considerada a experiência dos especialistas em reumatologia pediátrica e reabilitação do Lar Escola São Francisco e da Universidade Federal de São Paulo.

Resumo: os pacientes com AIJ podem apresentar dor e limitação da amplitude de movimento articular e conseqüente diminuição da capacidade física, com comprometimento das capacidades aeróbia e anaeróbia. Não só o comprometimento articular, mas as disfunções cardíacas e autonômicas colaboram nesse processo, tendo como conseqüência uma baixa capacidade de executar atividades esportivas e atividades de vida diárias (AVDs). O American College of Rheumatology recomenda 30 minutos de atividade com intensidade moderada de duas a três vezes por semana. A hidroterapia está relacionada a uma maior aderência ao tratamento, além de auxiliar na diminuição da percepção dolorosa e dificuldade apresentada na realização das AVDs. As outras modalidades de reabilitação, tais como massagem, educação, proteção articular, conservação de energia e órteses, também são discutidas nesta revisão. Conclusão: há poucos estudos na literatura sobre reabilitação em crianças com AIJ, especialmente no que se refere a temas como prescrição adequada de exercícios, cargas, número de séries e repetições, bem como qual a melhor opção a ser utilizada - solo ou piscina. Acreditamos que mais estudos científicos são necessários para que possamos prescrever adequadamente os diversos tipos de exercícios.

Palavras-chave: reabilitação, fisioterapia, artrite idiopática juvenil.

\begin{abstract}
Introduction and aims: juvenile idiopathic arthritis (JIA) may cause permanent physical disabilities in children and adolescents. This study aimed to describe the several kinds of rehabilitation procedures, ranging from evaluation to prescription of exercises, as well as the elaboration of a practical rehabilitation guide for JIA patients. Sources of data: the research was based on data from Medline and Lilacs. The opinion of experts working on the Pediatric Rheumatology service from Lar Escola São Francisco and Universidade Federal de São Paulo was considered on the debate of several topics. Summary: JIA patients may present pain and limitation of joint movement thereby leading to decrease in physical capacity, affecting both aerobic and anaerobic activities. In addition to the joint compromise, cardiac and autonomic dysfunctions collaborate on this process, impairing sport and everyday activities. The American College of Rheumatology recommends 30-minute activity with moderate intensity, two to three times weekly. Hydrotherapy is associated to treatment adherence, besides helping in decreasing pain perception and adding to cope with daily activities. Other rehabilitation modalities, such as massage, education, joint protection, energy conservation, and splints are also considered in the present review. Conclusion: there are few studies in the literature focusing on rehabilitation in children with JIA. Particularly, there is a lack of studies concerning aspects of adequate prescription of exercises, weight-bearing, number of series and repetitions, as well as the best choice regarding ground or water activity. We believe that additional information is needed in order to improve the physical care to these patients.
\end{abstract}

Keywords: rehabilitation, physical therapy, juvenile idiopathic arthritis.

\footnotetext{
Recebido em 23/10/06. Aprovado, após revisão, em 26/03/07.

Trabalho realizado na Disciplina de Alergia, Imunologia e Reumatologia do Departamento de Pediatria e na Disciplina de Reumatologia do Departamento de Medicina da Universidade Federal de São Paulo/Escola Paulista de Medicina (Unifesp/EPM).

1. Mestranda em Reabilitação da Disciplina de Reumatologia do Departamento de Medicina da Unifesp/EPM

2. Doutorando em Ciências da Saúde da Disciplina de Reumatologia do Departamento de Medicina da Unifesp/EPM.

3. Doutorando em Ciências da Saúde da Disciplina de Cardiologia do Departamento de Medicina da Unifesp/EPM

4. Mestranda em Neurociências do Departamento de Neurologia e Neurocirurgia da Unifesp/EPM.

5. Professor adjunto da Disciplina de Alergia, Imunologia e Reumatologia do Departamento de Pediatria da Unifesp/EPM.

6. Professora afiliada da Disciplina de Alergia, Imunologia e Reumatologia do Departamento de Pediatria da Unifesp/EPM.

7. Professor adjunto livre-docente da Disciplina de Reumatologia do Departamento de Medicina da Unifesp/EPM.

8. Professora associada e chefe do Setor de Reumatologia Pediátrica da Disciplina de Alergia, Imunologia e Reumatologia do Departamento de Pediatria - Unifesp.

Endereço para correspondência: Vanessa Cristina Bueno, Rua Borges Lagoa, 802, CEP 04038-001, São Paulo, SP, telefone: (11) 5574-7627, e-mail: vancris_bueno@hotmail.com.
} 
A artrite idiopática juvenil (AIJ) é uma doença de etiologia desconhecida que se caracteriza pela presença de artrite crônica e manifestações gerais e viscerais. Muitos pacientes podem apresentar incapacidade física permanente. O diagnóstico é essencialmente clínico e de exclusão e feito na presença de artrite em uma ou mais articulações por um período maior ou igual a seis semanas em crianças com idade inferior a 16 anos $^{(1,2)}$.

O quadro clínico pode ter início com sintomas e sinais constitucionais (anorexia, fadiga, perda de peso e diminuição no crescimento) e artrite (dor, calor, rubor, edema e perda funcional) em uma ou mais articulações - joelhos, tornozelos, punhos, cotovelos e quadril são as articulações mais acometidas; observam-se rigidez matinal e entesites. $\mathrm{O}$ rash (erupção cutânea macular fina e rósea) é comum em quadros sistêmicos, é raramente pruriginoso e localiza-se, preferencialmente, no tronco e nas porções proximais dos membros ${ }^{(1,2)}$.

A assistência ao paciente com AIJ baseia-se em um modelo multiprofissional, ou seja, além do uso de medicamentos (antiinflamatórios, drogas de base, imunossupressores e/ou agentes biológicos), são necessárias fisioterapia e/ ou terapia ocupacional. Ocasionalmente, são necessárias cirurgias para correção de deformidades, além de suporte psicológico ${ }^{(3,4)}$.

A avaliação da reabilitação consiste na análise minuciosa da integridade das articulações [amplitude de movimento $(\mathrm{ADM})$ - biomecânica articular], musculatura (força e trofismo), marcha, atividades de vida diárias (AVDs) e na aplicação de questionários para a obtenção de dados que podem ser utilizados para avaliação de melhora e posterior alta do paciente ${ }^{(3)}$. O questionário Escola Paulista de Medicina Range of Motion - Pediátrico (EPM Rom Pediátrico) foi validado em 1999 para ser utilizado em crianças com AIJ. Esse instrumento avalia a ADM de dez articulações bilateralmente - coluna cervical, ombros, punhos, polegar, quadril, joelhos e tornozelos. Possui uma escala que varia de 0 (sem limitação) a 3 (limitação intensa) - quanto maior sua pontuação, maior a limitação encontrada ${ }^{(5)}$. Outro instrumento utilizado na avaliação da capacidade física de crianças e adolescentes com AIJ é o Childhood Health Assessment Questionnaire (CHAQ), que foi traduzido e validado em 1994. É composto por 30 itens com os seguintes domínios: AVDs, levantar-se, alimentação, higiene pessoal, utilização dos membros superiores, preensão e atividades diárias, com respostas que variam de 0 (zero), sem qualquer dificuldade, a 3 (três), incapaz de realizar; maiores pontuações são indicativas de pior capacidade funcional ${ }^{(6)}$. O Pediatric Quality of Life 4.0 (PedsQl) foi desenvolvido em 2001 e encontra-se em processo de validação para nossa língua e cultura. Trata-se de um questionário genérico que avalia qualidade de vida de crianças e adolescentes de 2 a 18 anos. Consiste em 23 itens distribuídos em funções física, emocional, social e escolar. Utiliza-se, em cada uma de suas perguntas, uma escala de cinco pontos do tipo Likert scale, sua pontuação varia de 0 ("nenhuma dificuldade") a 4 ("muita dificuldade"), maiores pontuações são indicativas de pior função( ${ }^{(7)}$. O Child Health Questionnaire (CHQ) também é um questionário genérico de qualidade de vida e foi traduzido e validado para uso em nosso meio em 2001 para AIJ. O CHQ possui versões para crianças e pais. São avaliados os seguintes domínios: saúde global, atividades físicas, atividades diárias, dor, comportamento, bem-estar, auto-estima, um melhor estado de saúde ${ }^{(8)}$

As escalas visuais analógicas também são ferramentas úteis no seguimento dos pacientes com AIJ. Tanto profissionais quanto pacientes e pais podem preenchê-las a cada três a seis meses, no intuito de quantificar a melhora da atividade da doença (profissionais) ou piora do estado geral (pacientes e cuidadores).

\section{DISFUNÇÃO CARDIOVASCULAR NA AIJ}

Algumas crianças com AIJ podem apresentar baixa performance física, com comprometimento das capacidades aeróbia e anaeróbia, levando a uma baixa capacidade de executar atividades esportivas e AVDs s ${ }^{(9)}$. Portanto, é necessária uma avaliação adequada para a realização da reabilitação. O comprometimento articular, as disfunções cardíacas e as autonômicas colaboram para a reduzida capacidade cardiovascular ${ }^{(10-12)}$. $\mathrm{O}$ volume de oxigênio $\left(\mathrm{VO}_{2}\right)$ máximo é uma variável representativa da capacidade física, está diretamente correlacionado com a qualidade de vida e, freqüentemente, encontra-se diminuído em pacientes com $\mathrm{AIJ}^{(12,13)}$.

O exercício aeróbio é caracterizado pela utilização de grandes grupos musculares, como caminhada, corrida, natação e ciclismo, que são realizados de forma rítmica, com intensidade de $60 \%$ a $85 \%$ da freqüência cardíaca máxima, por um longo período e, conseqüentemente, predomínio do metabolismo oxidativo. É recomendado como o exercício mais eficiente para a melhora da capacidade cardiovascular e, conseqüentemente, aumento do $\mathrm{VO}_{2}$ máximo ${ }^{(14,15)}$.

$\mathrm{O}$ American College of Rheumatology (ACR) recomenda treinamento físico por meio do exercício aeróbio para pacientes adultos com artrite reumatóide (AR) (60\% a 85\% da freqüência cardíaca máxima de intensidade), com duração de 30 a 60 minutos, duas a três vezes por semana. A recomen- 
dação para crianças com AIJ é de 30 minutos de atividade com intensidade moderada, três vezes por semana ${ }^{(16)}$. Cabe ressaltar que crianças apresentam menor volume sistólico, débito cardíaco, capacidade ventilatória ${ }^{(16)}$, menor concentração e atividade da enzima fosfofrutoquinase ${ }^{(17)}$, levando à necessidade de ajustes específicos na prescrição da intensidade do exercício aeróbio quando comparado ao adulto. Dessa forma, recomenda-se uma intensidade entre $50 \%$ e $70 \%$ da freqüência cardíaca de reserva ${ }^{(18,19)}$.

Pacientes com AIJ podem apresentar lesões articulares e/ou musculares, portanto toda prescrição de exercícios (aeróbios ou anaeróbios) deve ser realizada sob orientação de um profissional.

\section{ASPECTOS DA MUSCULATURA ESQUELÉTICA E TREINAMENTO RESISTIDO}

A força muscular apresenta correlação com a capacidade de realizar as AVDs ${ }^{(20)}$. Muitos pacientes com AIJ apresentam redução da força quando comparada à de indivíduos saudáveis ${ }^{(21)}$.

O exercício resistido tem se mostrado um eficiente recurso no processo de reabilitação das disfunções musculares, como perda da força. Um estudo realizado em pacientes com AR submetidos a um treinamento resistido com baixa carga e alta repetição mostrou redução na intensidade da dor nas articulações, melhora da força, da funcionalidade e do tempo total de permanência no teste ergométrico, além de significativa melhora na força, funcionalidade e na qualidade de vida ${ }^{(21)}$. O ACR indica exercícios resistidos para pacientes com AR de duas a três vezes na semana com $50 \%$ a $80 \%$ do teste de "I Repetição Máxima" (IRM), com uma ou duas séries de oito a 12 repetições. Crianças com AIJ devem fazer exercícios resistidos três vezes por semana para melhora da força e da resistência muscular. Entretanto, essa recomendação se mostra superficial diante das particularidades do organismo da criança e do adolescente ${ }^{(16)}$. A redução da força muscular, a instabilidade articular e a redução da densidade óssea observadas na criança com AIJ justificam a utilização dos exercícios resistidos. Todavia, a escassez de estudos dificulta a prescrição detalhada de cargas, número de séries e repetições ${ }^{(21,22)}$.

\section{HIDROTERAPIA NA AIJ}

$\mathrm{O}$ uso da hidroterapia como terapêutica nas doenças reumatológicas iniciou-se com Hipócrates (460-375). O entendimento dos princípios físicos e da termodinâmica da água proporciona o seu uso racional. A flutuação permite ao paciente caminhar com pouca sobrecarga articular devido à redução dos efeitos da gravidade e ao conseqüente aumento da amplitude de movimento articular. A pressão hidrostática exerce um efeito positivo, durante a imersão (a mesma pressão é exercida em todas as direções do corpo e aumenta com a profundidade), de diminuição de edemas, pois o sistema venoso redistribui o sangue das extremidades para o tórax. A resistência da água pode ser utilizada passiva e ativamente; movimentos passivos através da água facilitam o relaxamento e alongamento de tecidos moles; ativamente, a resistência da água aumenta o gasto energético necessário para movimentar as extremidades, promovendo fortalecimento muscular. A termodinâmica também deve ser levada em consideração e está relatada com a capacidade de o corpo submerso trocar energia (calor) com a água por meio da condução e convecção, tendo efeito positivo sobre tecidos moles que podem facilmente ser mobilizados, aumentando o grau de movimento e diminuindo a dor articular. Os benefícios proporcionados pela água são: relaxamento muscular, aumento do fluxo sanguíneo e da flexibilidade, fortalecimento muscular, reeducação da marcha, melhora do equilíbrio e da coordenação e, finalmente, é uma atividade lúdica e de recreação ${ }^{(23-27)}$.

Templeton et al. ${ }^{(28)}$, estudando os efeitos da terapia aquática na flexibilidade articular e capacidade funcional em pacientes com doenças reumáticas, observaram um decréscimo na percepção de dor e diminuição da dificuldade apresentada na realização das AVDs.

Stenstrom et al.$^{(29)}$ seguiram por quatro anos dois grupos de 30 pacientes com diagnóstico de AR. Trinta pacientes realizaram treinamento dinâmico intensivo na água, com freqüência de uma vez por semana durante 40 minutos; o outro grupo não recebeu nenhum treinamento. Ao final do estudo, os pacientes tratados apresentavam aumento na força muscular e nos níveis de atividade física.

Bacon et al. ${ }^{(30)}$ estudaram os efeitos da hidroterapia em pacientes com AIJ e comprometimento de membros inferiores com idade entre 4 e 13 anos. O protocolo de treinamento foi de seis semanas e observou-se aumento significativo na $\mathrm{ADM}$ de rotações interna e externa de quadril bilateralmente, assim como aumento da força muscular. Takken et al. ${ }^{(3)}$ realizaram um estudo-piloto em dez meninas com AIJ na tentativa de observar se o programa de treinamento aquático aeróbico ( 15 sessões de 60 minutos cada uma, uma vez por semana, durante 15 semanas) poderia aumentar não somente a resistência, mas também a capacidade funcional. Ao final do tratamento, foi observado aumento nas medidas de avaliação, entretanto sem diferença estatística. Nesse estudo, a aderência foi de $85 \%$. Os mesmos autores avaliaram os efeitos de 
um programa de treinamento aquático em 54 crianças com AIJ, randomizadas em dois grupos: controle e tratamento com um programa de treinamento supervisionado de 20 sessões uma vez por semana, com duração de uma hora. Ao final do treinamento, não foram encontradas diferenças estatisticamente significantes entre os dois grupos. Takken et al. ${ }^{(31)}$ sugerem que os exercícios sejam realizados com uma freqüência maior do que três vezes por semana. Epps $e t$ $a l^{(32)}$ estudaram pacientes com AIJ e compararam os efeitos da combinação da hidroterapia com a fisioterapia convencional em solo e somente fisioterapia em solo com o intuito de avaliar o custo-benefício da hidroterapia. O custo foi calculado de acordo com o número de faltas ao trabalho dos pais. Cem pacientes faziam parte do grupo combinado e cem, do grupo solo. Os pacientes de ambos os grupos receberam tratamento durante dois meses, duas vezes por semana com duração de uma hora cada sessão. Após dois meses de intervenção, não foram observadas diferenças estatisticamente significantes relacionadas ao custo nos dois grupos, também não houve exacerbação da doença em nenhum paciente, indicando que ambos os tratamentos são seguros.

\section{EDUCAÇÃO DO PACIENTE COM AIJ}

A educação do paciente com doença crônica e de seus familiares é parte importante no tratamento e está associada a uma melhora na compreensão da doença e um aumento na aderência ao tratamento ${ }^{(33)}$.

De um modo geral, os adolescentes têm dificuldade em assumir seu próprio cuidado. São necessários reforços positivos e orientações direcionadas para uma melhor compreensão de sua doença ${ }^{(34)}$.

Para que um programa de educação seja efetivo é importante detectar as necessidades individuais de cada paciente, principalmente dos adolescentes ${ }^{(35,36)}$. Um estudo realizado com adolescentes asmáticos mostrou que, em 98\% dos casos, os pacientes que receberam orientações (como lidar com a doença) tiveram melhor aderência ao tratamento quando comparados ao grupo que não recebeu as orientações ${ }^{(37)}$.

Os adolescentes com asma, epilepsia, diabete melito e AIJ esperam do educador habilidade para criar um bom ambiente para o tratamento, conhecimento do assunto, facilidade na comunicação e habilidade em motivá-los a aprender ${ }^{(36)}$.

Crianças com AIJ e seus pais têm grande interesse em ter acesso à informação, visando a uma melhor compreensão da doença. Deve ser dada ênfase ao aspecto social e emocional da doença; e grupos de educação, grupos informais de suporte, atividades em finais de semana e alojamentos de verão (mais comuns nos Estados Unidos) podem ser acolhedores, reduzindo o isolamento ${ }^{(38)}$.

Em 2001, Manuel ${ }^{(39)}$ observou alterações psicológicas em mães de crianças com AIJ. O elevado nível de estresse psicossocial prediz aumento nos sintomas emocionais, como depressão e ansiedade. Uma intervenção que vise à educação das mães pode diminuir o efeito do estresse maternal.

Bruns, em 2003 ${ }^{(40)}$, avaliou os aspectos da qualidade de vida que são mais comprometidos nos cuidadores primários (principal pessoa responsável pela assistência do paciente), observando, portanto, que os aspectos emocionais tiveram maior significância e que o impacto nos cuidadores de pacientes com AIJ depende mais dos aspectos emocionais dos próprios cuidadores do que das condições físicas do paciente.

Um programa educacional para pacientes com artrite idiopática juvenil e seus pais deve conter as seguintes orientações $^{(41)}$ :

1. Aspectos psicossociais;

2. Aspectos do tratamento: uso da medicação de maneira adequada;

3. Aspectos físicos: programa de exercício terapêutico e exercício físico;

4. Terapêutica no controle da dor;

5. Avaliação do ambiente escolar, tanto no aspecto de compreensão dos colegas e professores quanto no aspecto arquitetônico;

6. Transição da fase de criança/adolescente para fase adulta (transitional care).

O programa educacional tem que fornecer informações aos pacientes e a seus pais sobre "como enfrentar" a doença, objetivando aumentar a aderência ao tratamento e melhorar a qualidade de vida.

Muitos adolescentes com AIJ mantêm o tratamento em clínicas pediátricas $^{(42)}$. Há necessidade de serviços de transição dentro da reumatologia de adultos, com ênfase em um processo que englobe as necessidades médicas, psicossociais, educacionais e vocacionais para os adolescentes que iniciaram a doença na infầncia e estão chegando à fase adulta ${ }^{(43)}$.

Um programa de cuidado transicional foi identificado como sendo um importante apoio para adolescentes e pais. Requer o envolvimento de equipe multiprofissional constituída por reumatologistas, enfermeiros, fisioterapeutas, terapeutas ocupacionais e psicólogos. O programa deve conter: informação sobre a doença; equipe de profissionais especializados; autonomia no cuidado da saúde; independência na vida diária; orientação sobre puberdade; educação 
e orientação vocacional; saúde bucal; exercício; drogas e álcool; orientação sexual; e diferença entre reumatologia pediátrica e reumatologia do adulto ${ }^{(44)}$.

O programa de transição parece ter boa aceitação pelos adolescentes, seus pais e profissionais da saúde $\mathrm{AIJ}^{(45)}$.

\section{MASSAGEM}

Foi encontrado um único estudo não-randomizado que sugere que a massagem pode servir como recurso terapêutico para crianças com AIJ. Foram selecionados 20 pacientes com AIJ e divididos em dois grupos: o primeiro recebeu massagem aplicada pelos pais por 15 minutos, diariamente, por 30 dias; e o segundo recebeu sessões de relaxamento por 15 minutos, todas as noites, por 30 dias. A massagem foi dividida em duas etapas: primeiro, os pacientes permaneciam em decúbito dorsal e posteriormente ventral, com movimentos circulares e deslizamentos superficiais e profundos; todos os pacientes foram massageados. Foi utilizado um óleo específico para massagem, na seguinte ordem: face, abdômen, membros inferiores (quadril aos dedos dos pés) e membros superiores (ombros aos dedos das mãos). Foram avaliados depressão, ansiedade, dor, nível de cortisol salivar e o número de articulações ativas. Os pacientes que receberam massagem apresentaram diminuição da ansiedade, depressão, nível de cortisol salivar, dor e melhora na realização das atividades quando comparados com o grupo de relaxamento ${ }^{(45)}$.

\section{ADAPTAÇÕES, PROTEÇÃO ARTICULAR, CONSERVAÇÃO DE ENERGIA E ÓRTESES}

A literatura tem poucas referências sobre adaptações em AIJ (independência nas atividades de vida diária e órteses). Em um estudo, foram coletadas informações sobre diferentes centros de reumatologia pediátrica nos Estados Unidos, no Canadá e no Reino Unido. Os autores concluíram que a fisioterapia e a terapia ocupacional são fundamentais no tratamento da $\mathrm{AIJ}^{(46)}$.

Em uma revisão sistemática sobre terapia ocupacional em pacientes com artrite reumatóide, concluiu-se que existe evidência da orientação de proteção articular nesses pacientes, porém não há trabalhos sobre essa intervenção em $\mathrm{AIJ}^{(47)}$.

Há poucos estudos na literatura sobre reabilitação em crianças com AIJ, especialmente no que se refere a temas como prescrição adequada de exercícios, cargas, número de séries e repetições, bem como qual a melhor opção a ser utilizada - solo ou piscina. Acreditamos que mais estudos científicos são necessários para que possamos prescrever adequadamente os diversos tipos de exercícios.

\section{GUIA PRÁTICO PARA UM PROGRAMA DE REABILITAÇÃO PARA PACIENTES COM AIJ - BASEADO NA MELHOR EVIDÊNCIA CIENTÍFICA E NA PRÁTICA CLÍNICA DIÁRIA}

Uma avaliação fisioterapêutica deve conter:

1. Anamnese

2. Exame físico:

a. Inspeção

b. Palpação

3. Goniometria

4. Avaliação da força muscular

a. Dinamometria de mão e dedos

i. PINCH (mão)

ii. Jamar

b. Aparelho isocinético

5. Perimetria da articulação acometida

6. Avaliação de edema (caso haja)

a. Volúmetro

7. Questionários
a. CHAQ - capacidade funcional
b. Pediatric EPM ROM - amplitude de movimento
c. PedsQL ou CHQ - qualidade de vida
d. Escala visual analógica - dor

8. Marcha
a. Auxiliares
b. Biomecânica

9. Prescrição de exercícios
a. Alongamentos livres e assistidos
b. Exercícios assistidos e ativos
c. Exercícios com carga ou resistência, com cautela devido à baixa evidência científica

10. Educação ao paciente e familiares

11. Conservação de energia
a. Planejar
b. Priorizar as tarefas
c. Delegar

12. Proteção articular

a. Órteses: posicionamento noturno, funcional de punho, botoeira, pescoço de cisne, desvio ulnar e canaletas

b. Palmilhas

c. Dispositivos de marcha: andador, bengala e muletas (axilar e canadense)

d. Adaptações: para a realização das atividades de vida prática e diária

Declaramos a inexistência de conflitos de interesse. 


\section{REFERÊNCIAS}

1. Kullas DT, Schanberg L: Juvenile idiopatic arthritis. Curr Opinion in Rheumatology 13:399-404, 2001.

2. Petty RE, Southwood TR, Baum J, et al.: Revision of the proposed classification criteria for juvenile idiopatic Arthritis: Durban, 1997. Rheumatol 25:1991-4, 1998.

3. Takken T, Van der Net J, Kuis W, Helders PJM: Do juvenile idiopathic patients benefit from na exercise program? A pilot study. Arthritis Rheum 45:81-5, 2001.

4. Hashkes PJ, Laxer RM: Medical treatmente of juvenile idiopatic arthritis. JAMA 294:1671-84, 2005.

5. Len C, Ferraz MB, Goldenberg J, et al.: Pediatric Escola Paulista de Medicina range of motion scale: a reduce joint count scale for general use in juvenile rheumatoid arthritis. J Rheumatol 26:909-13, 1999.

6. Len C, Goldenberg J, Ferraz MB, Hilário MO, Sacchetti S: Crosscultural reability of the childhood health assessment questionnaire. J Rheumatol 21:2349-52, 1994.

7. Varni JW, Seid M, Kurtin PS: The pedsQL 4.0: Reability and validity of the pediatric quality of life inventory version 4.0 generic core scales in healthy and patient populations. Med Care 39:800-12, 2001.

8. Machado CS, Ruperto N, Silva CH, et al.: Paediatric Rheumatology International Trials Organization. The Brazilian version of the Childhood Health Assessment Questionnaire (CHAQ) and the Child Health Questionnaire (CHQ). Clin Exp Rheumatol 19:S23:S25-29, 2001.

9. Takken T, Hemel A, Van der Net J, Helders PJM. Relationship between functional ability and physical fitness in juvenile idiopathic patients. Scand J Rheumatol 32:174-8, 2003.

10. Takken T, Hemel A, Van der Net J, Helders PJM: Aerobic fitness in children with juvenile idiopathic arthritis: a systematic review. J Rheumatol 29:2643-7, 2002.

11. Bernstein B, Takahashi M, Hanson V: Cardiac involvement in juvenile rheumatoid arthritis. J Pediatr 85:313-7, 1974.

12. Bharti BB, Kumar S, Kapoor A, Agarwal A, Mishra R, Sinha N: Assessment of left ventricular systolic and diastolic function in juvenile rheumatoid arthritis. J Postgrad Med 50:262-7, 2004.

13. Kuis W, de Jong-de Vos van Steenwijk C, Sinnema G, et al.: The autonomic nervous system and the immune system in juvenile rheumatoid arthritis. Brain Behav Immun 10:387-98, 1996.

14. NIH Consensus Development Panel on Physical Activity and Cardiovascular Health. Physical activity and cardiovascular health. JAMA 276:241-6, 1996.

15. Ward A, Malloy P, Rippe J: Exercise prescription guidelines for normal and cardiac populations. Cardiol Clin 5:197-210, 1987.

16. Minor M, Stenstrom CH, Klepper SE, Hurley M, Ettinger WH: Work Group Recommendations: 2002 Exercise and Physical Activity Conference, St. Louis, Missouri Session V: Evidence of Benefit of Exercise and Physical Activity in Arthritis Arthritis Rheum 49:453-4, 2003.

17. Rowland TW: Developmental aspects of physiological function relating to aerobic exercise in children. Sports Med 10:255-66, 1990.

18. Malina RM: Physical activity and training: effects on stature and the adolescent growth spurt. Med Sci Sports Exerc 26:759-66, 1994.
19. Klepper SE, Giannini MJ: Physical conditioning in children with arthritis: Assessment and guidelines for exercise prescription. Arthritis Care Res 7:226-36, 1994.

20. American College of Sport Medicine. ACM's Guidelines for exercise testing and prescription. $6^{\mathrm{a}} \mathrm{ed}$. Baltimore, Lippincott Williams \& Wilkins, 2000

21. Giannini MJ, Protas EJ: Comparison of peak isometric knee extensor torque in children with and without juvenile rheumatoid arthritis. Arthritis Care Res 6:82-8, 1993.

22. Komatireddy GR, Leitch RW, Cella K, Browning G, Minor M: Efficacy of low load resistive muscle training in patients with rheumatoid arthritis functional class II and III. J Rheumatol 24:1531-9, 1997.

23 Cunha MCB, Labronicci RHDD, Oliveira ASB, Gabbai AA: Hidroterapia. Fisioterapia Brasil 2:379-85, 2001.

24. Cardoso JR, Atallah NA, Cardoso APRG, et al.: Aquatic Therapy exercise for trearing rheumatoid arthritis [protocol]. Cochrane Review Issue 3, 2204.

25. Napoletan J: An innovation in aquatic therapy: the underwater treadmill. Physical Therapy 7:43-4, 1994

26. Golland A: Basic Hydrotherapy. Physiotherapy 67(9):258-62, 1981

27. Geigle PR, Cheek WL, Gould ML, Hunt HC. Aquatic physical therapy for balance: the interaction of somatosensory and hydrodynamic principles. The Journal of Aquatic Physical Therapy 5:4-10, 1997.

28. Templeton MS, Booth DL, O'Kelly WD: Effects of aquatic therapy on joint flexibility and functional ability in subjects with rheumatic disease. JOSPT 23:376-81, 1996.

29. Stenstrom CH, Lindell B, Swanberg E, Swanberg P, HarnsRindgahl K, Nordemar R: Intensive dynamic training in water for rheumatoid arthritis functional class II - a long-term study of effects. Scand J Rheunatol 20:358-65, 1991.

30. Bacon MC, Nicholson C, Bincler H, White PH: Juvenile rheumatoid arthritis. Aquactic exercise and lower-extremity function. Arthritis Care Res 4:102-5, 1991.

31. Takken T, Van der Net J, Kuis W, Helders PJM: Aquatic fitness training for children with juvenile idiopathic arthritis. Rheumatology 42:1408-14, 2003.

32. Epps H, Ginnelly L, Utley M, et al.: Is hydrotherapy cost-effective? A randomised controlled trial of combined hydrotherapy programmes compared with physicaltherapy land techiniques in children with juvenile idiopathic arthritis. Health Technol Assess 9:3-10, 2005.

33. Klein JD, Allan MJ, Elster AB, et al.: Improving adolescents preventive care in community health centers. Pediatrics 107 376-83, 2001

34. Carson AR, Voorhees HK: Adult pediatric patients. Am J Nursing 101:46-55, 2001

35. Divertc V: Strategies to promote medication adherence in children with asthma. Am J Maternal Child Nurs 27:10-8, 2002.

36. Kyngäs H: Patient education: perspective of adolescents with a chronic disease. J Clin Nurs 12:744-51, 2003.

37. Kyngäs $\mathrm{H}$ : Compliance of adolescents with asthma. Nursing and Health Sci 1:195-202, 1999. 
38. Barlow JH, Shaw KL, Harrison K: Consulting the 'experts': children's and parents' perceptions of psycho-educational interventions in the context of juvenile chronic arthritis. Health Educ Res 14:597-610, 1999.

39. Manuel JC: Risk and resistence factors in the adaptation in mothers of children with juvenile rheumatoid arthritis. J Pediatr Psycol 26:237-46, 2001.

40. Bruns A: Avaliação da qualidade de vida e do impacto da doença nos cuidadores primários de pacientes com artrite reumatóide juvenil. [mestrado] Universidade Federal de São Paulo, São Paulo, 2003.

41. Akikusa JD, Allen RC: Reducing the impact of rheumatic diseases in childhood. Best Practice \& Res Clin Rheumatol 16:333-45, 2002.

42. Packham JC, Hall MA: Long-term follow-up of 246 adults with juvenile idiopathic arthritis: education and employment. Rheumatology 41:1436-9, 2002.

43. Blum RW, Garell D, Hadgman $\mathrm{CH}$, et al.: Transition from child-centred to adult health systems for adolescents with chronic conditions. A position paper of the Society for adolescent medicine. J Adolesc Health 14:570-6, 1993.

44. McDonagh JE, Shaw KL, Southwood TR: Growing up and moving on in rheumatology: development and preliminary evaluation of a transitional care programme for multicentre cohort of adolescents with juvenile idiopathic arthritis. J Child Health Care 10:22-42, 2006.

45. Field T, Hernandez-Reif M, Seligman S, Krasnegor J, Sunshine W: Juvenile rheumatoid arthritis: Benefits from massage therapy. J Pediatr Psychol 22:607-17, 1997.

46. Hackett J, Johnson B, Parkin A, Southwood T: Physiotherapy and occupational therapy for juvenile chronic arthritis: custom and practice in five centres in the UK, USA and Canada. Br J Rheumatol 35:695-9, 1996.

47. Steultjens EMJ, Dekker J, Bouter LM, van Schaardenburg D: Occupational therapy for rheumatoid arthritis. Cochrane Database Syst Rev (1):Cd003114, 2004. 